\title{
A Compression based Energy Efficient Protocol for Mobile Cloud Computing
}

\author{
D Satyanarayana, Abdullah Said Al Kalbani
}

\begin{abstract}
: the demands of the present society transformed the world from $4 G$ to $5 G$ communication system. One of the primary focus of $5 G$ technology is cloud computing, where the end users can get the unavailable services from the cloud. An exceptional type of cloud computing is Mobile Cloud Computing (MCC), where the end users are mobile devices. In the MCC, the resources at the mobile device are constrained or limited. The energy consumption during communication process of MCC is directly proportional to the amount of the data to be transmitted. In this paper, we propose a new method to reduce the energy consumption in the MCC. The simulation results consolidate the claim.
\end{abstract}

Keywords: 5G communication, cloud computing, mobile cloud computing, energy consumption.

\section{INTRODUCTION}

Nowadays, the cloud computing has become popular because of the increase in service oriented network infrastructures, rather than full-fledged systems. There are huge number of industries getting the benefits from the cloud computing technology. However, this technology has many challenges and issues to solve, and it motivates various research areas to improve the system. The National Institute of Standards and Technology (NIST) presented the definition of cloud computing as a model for enabling ubiquitous, convenient, and on-demand network access to a shared pool of configurable computing resources (e.g., networks, servers, storage, applications, and services) that can be rapidly provisioned and released with minimal management of effort or service provider interaction [1]. A special kind of cloud computing is called mobile cloud computing, where the service-requesting device is the mobile device. In the mobile cloud-computing environment, the resources at the mobile side are constrained or limited. One of the precious resources in mobile devices is battery power. The energy consumption in $M C C$ is directly affects the battery power in mobile client. Generally, the considerable energy consumption in $M C C$ occurs in two contexts, during the communication process and the computation process. However, the communication energy consumption is significantly higher than the computational energy consumption. In this paper, we primarily focus on reducing energy consumption during the

Revised Manuscript Received on September 22, 2019.

Degala Satyanarayana, College of Engineering, University of Buraimi, Oman.

Abdullah Said Al Kalbani, College of Engineering, University of Buraimi, Oman. communication process. In the $M C C$, the mobile clients get the services from the cloud by sending the required information to the cloud. The more information is transmitted the more energy is consumed. In this paper, we investigate and propose a new energy efficient $M C C$ methodology called Energy efficient Mobile Cloud Computing (EMCC). This method reduces the energy consumption in the resource-constrained systems without losing the functionality of $M C C$.

The reminder of this paper is organized as follows: The section II describes the related work and the section III gives more details on the proposed methodology. The section IV describes the simulation work and the results. Finally, the conclusion is given in the section V.

\section{RELATED WORK}

Cloud computing is a new way of providing computing resources and services. It refers to an on-demand infrastructure that allows users to access computing resources anytime from anywhere. The cloud computing offers to users and business three main advantages: (a) enormous computing resources available on demand, (b) payment for use as needed, and (c) simplified IT management and maintenance capabilities [1].

The cloud computing offers a wide variety of services and resource by using the internet. The users get the resources such as software, storage, servers, and the network services whenever required to use them for their applications [2]. Through cloud computing the services are scalable to the real world. Since the cloud computing provides virtual services, which are available based on the user request, both the cloud users and cloud providers get the considerable economic advantages. In last few years, the cloud computing is more interested by mobile users. However, for many mobile users, the motivation of using cloud computing is different from the motivation of PC-based users. In other words, the requirements of mobile users are varied with the requirements of PC-based users.

The mobile cloud computing can be seen as a bridge that fills the gap between the limited computing resources of Smart Mobile Device (SMD) and processing requirements of intensive applications on $S M D$. It has attracted the attention of business people as a beneficial and useful business solution that minimizes the development and execution costs of mobile applications, allowing mobile users to acquire latest technology conveniently on an on-demand basis [3]. 
The $M C C$ promises several benefits such as extra battery life and storage, scalability, and reliability. However, there are still challenges that must be addressed in order to enable the ubiquitous deployment and adoption of mobile cloud computing. Some of these challenges include security, privacy and trust, bandwidth and data transfer, data management and synchronization, energy efficiency, and heterogeneity [4].

The mobile cloud-computing paradigm provides the required support for the creation of cyber-physical systems, which may be used to improve the daily life experience of citizens as well as to bring social and economic benefits. Mobile networks are suffering from "capacity crunch", meaning that network providers are struggling to meet the demand of mobile data services. To provide Quality-of-Service $(Q o S)$ to the user, the network provider must provide a rich set of services such as, increasing the network capacity and energy efficiency based on the user's mobility patterns [5].

Due to the limited life of battery power in mobile devices, the optimization of device's resources and its power consumption allows for maximum usage of the device [6]. There are many researchers gave different solutions in this area. In [7], the author provides a theoretical framework of energy-optimal mobile cloud computing under stochastic wireless channel. The researchers tried to conserve energy for the mobile device by optimally executing mobile applications in the mobile device or offloading to the cloud.

The author Gai. et. al [8] proposed a dynamic energy-aware cloudlet-based mobile cloud-computing model $(D E C M)$ focusing on solving the additional energy consumptions during the wireless communications by leveraging dynamic cloudlets (DCL)-based model.

A variation of mobile cloud computing called virtualized cloud systems, the mobile entity can either be a physical mobile device or cloud software agent. A software agent is a piece of code as an agent for the user or another program and they offer benefits to the end user by automating repetitive tasks. In the present cloud environment, the software agent provides benefits of migrating or composing of software codes. The service parameters for this type paradigm includes sharing of code, synchronization with other entities, information caching capabilities, networking and communication connectivity, the real time presence, and the delay parameter. The above capabilities create a new network on top of existing physical network. In other words, the virtual entities are mapped on the physical systems, which extends the functionalities and capabilities. An active research is being carried out to optimize the interaction between cyber physical systems (CPS) and cyber virtual systems (CVS). The CPS is designed using mobile and smart entities, whereas $C V S$ is designed using virtual resources and services. The author Huang et al. described these transformations [9].

In $M C C$, the energy is primary concern as it is a limited resource in mobile entity. One of the method for saving the energy focuses on off-loading the computation on the mobile

client that exceeds the energy cost of additional communication. It has been described comprehensively about the various methods and their implications on energy efficiency in mobile cloud computing [10].

The number of applications and the services are increased for mobile devices due to the emerging of mobile cloud computing. The user experiences the effect of battery power in the mobile devices due to the increasing of the applications. The Location Based Application $(L B A)$ is one heavily used in the mobile applications. The Global Positioning Systems (GPS) is an example of the $L B A$ and it presents limitations on surrounding energy [11].

In order to provide $M C C$ more convenient and ubiquitous characteristics, the cloud is provided powerful computing and the storage resources and reducing the front-end investment. Apart from the benefits, the $M C C$ has many challenges such as $Q o S$ guarantee, mobility management, and security issues. Among these issues, the important challenge is energy efficiency in the mobile devices, since the battery industry is moving slowly [11].

The energy consumed by the mobile devices in each $M C C$ model is mainly composed of:

1. Computing energy: This energy is consumed by the mobile device for the execution of local services. This energy depends on both the mobile characteristics (computing and battery features) and the performed computing.

2. Communication energy: This is consumed during transmission and reception of data through communication networks. This energy depends on the mobile characteristics, the network characteristics, and the size of data that is transferred. The table I shows the consumed energy measured for each tested solution [12].

Table- I: Consumed energy for program execution.

\begin{tabular}{|l|c|c|l|}
\hline Solution & $\begin{array}{l}\text { Execution } \\
\text { Time }(\mathrm{ms})\end{array}$ & $\begin{array}{l}\text { Mobile } \\
\text { Capacity(mAH) }\end{array}$ & $\begin{array}{l}\text { Consumed } \\
\text { Energy Joules }\end{array}$ \\
\hline OSGi & 30 & 1440 & 0.1596 \\
\hline Elijah & 79 & 1440 & 0.42028 \\
\hline Docker & 30 & 1440 & 0.1596 \\
\hline
\end{tabular}

The data compression is used in the proposed work, as it is required during the transmission of data between mobile and the cloud. The more data transmits, the more energy is consumed. Hence, compressing the data to be transmitted is a useful process. The summary of the data compression mechanisms are provided in [13]-[17].

\section{Contribution}

In the mobile cloud computing, the mobile devices use the services from the cloud. As the mobile devices are not connected to continuous power supply, the lifetime of the mobile devices is limited and considered a key research area. In other words, the battery power of the mobile device need to be considered a scarce resource in the MCC. In this section, 
we present a new energy efficient method for mobile cloud computing.

The basic idea of the proposed method is shown Fig. 1. The energy required for communication is directly proportional to the data to be transmitted during communication process of MCC. So, reducing the data to be transmitted leads to the reducing the energy consumption.

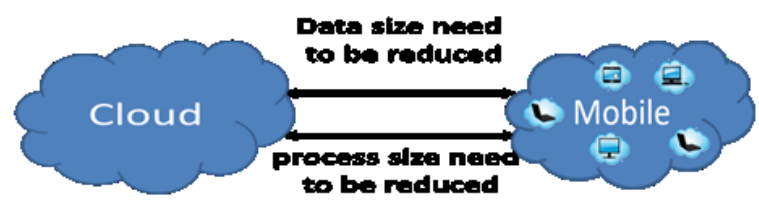

Fig. 1. Proposed method for saving energy in $M C C$

$$
\mathrm{E}=\alpha \mathrm{D}
$$

Where $E=$ the energy required for communication $D=$ the size of data

Hence, the proposed model aims to reduce the energy consumption during the $M C C$ processes. The proposed method considered two cases. In the case 1 , the data to be transmitted between the mobile device and the cloud need to be reduced. In case 2, the size of the process need to be reduced. By considering the case 1 and case 2, the proposed work can reduce the energy consumption significantly.

The algorithm for the proposed method is given in Algorithm I.

\section{Algorithm I: EMCC}

Step1: Request to the cloud for processing of data by mobile device. The cloud responds as accept /reject, see the Fig. 2. Step2: If the cloud accepts, compress the data using the Algorithm II and transmit the compressed data.

Step3: After processing the data, the result will be compressed using Algorithm II and send back to the mobile device from the cloud.

Step4: Request to the cloud by mobile device for processing the computation. The cloud responds to mobile by accept/reject, see the Fig. 3.

Step5: If the cloud accepts, use the Algorithm II to reduce the size of computation and send it to the cloud.

Step6: Once the cloud finishes the processing of computation, the compressed results will be sent back to the mobile device.

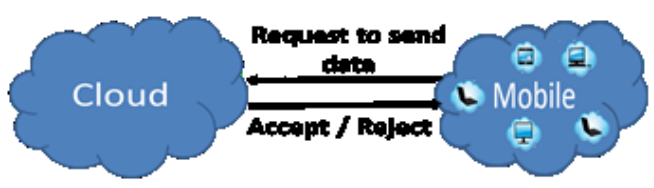

Fig. 2. Method for sending data in MCC

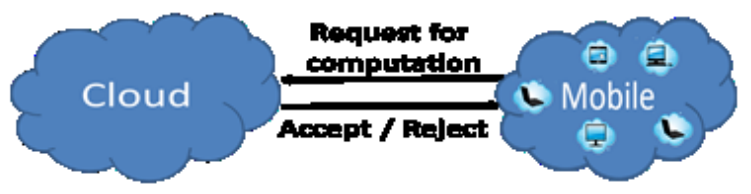

Fig. 3. Method for sending computation in MCC

In case I, the mobile device requesting the data from the cloud. This data could be a normal text, image, video, or an audio. In case II, the mobile device requesting the cloud for computational work. For example, the unavailability of a $\mathrm{C}++$ compiler in the mobile device, the mobile device can able to run a $C++$ program in the cloud. In both the cases, compressing the information can reduce the energy consumption in mobile device.

The compression process for the EMCC is presented in Algorithm II.

\section{Algorithm II: Compression Procedure}

Step1: If the data to be compressed is text, program, or software then go to the Step2, otherwise go the step3.

Step2: If the file has more data redundancy then use the Run Length Encoding ( $R L E$ ) compression method, otherwise use Huffman Encoding Compression (HEC) method and go the step 4.

Step3: If its video streaming, $D V D$ video, audio, or image files, then use lossy compression methods Discrete Cosine Transform $(D C T)$ or Discrete Wavelet Transform $(D W T)$ for the compression.

Step4: Calculate the Compression Factor $(C F)$. The $C F=$ Size of input bits/Size of output bits.

Step5: If the $C F \leq 1$ then transmit the data without any compression.

Step6: If ( $C F>C F \_$Thresh) then transmit the data with compression

Step7: If $(C F>1) \& \&\left(C F \leq C F \_\right.$Thresh $)$then estimate the communication cost (CC) and the decompression cost (DC). If $(D C>C C)$ then transmit without compression, else transmit with compression.

In the compression procedure, the data is compressed when the compression factor is more than one. In other words, we transmit the compressed data only if there is some advantage from the compression process during the data transmission, otherwise, the data is transmitted without any compression. Here, the CF_Thresh is the threshold on the compression size and it assures that the compression is definitively an advantage to the proposed protocol. Sometimes, the compressed data is between 1 and $C F \_$Thresh. In this case, we need to check the decompression cost $(D C)$ and the communication cost $(C C)$. Here, the $D C$ describes the energy required for the decompression process and $C C$ is the energy required for transmitting and receiving the data between the cloud and mobile entity. If the decompression cost is more than the communication cost then no need to compress, and transmit the information

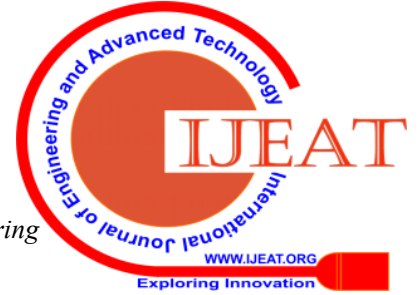


without compression. This activity will save the energy required for the decompression. In addition to this, the latency of whole process can also be reduced,

as there is no decompression process. However, there is a tradeoff between the latency and energy saving. We need to balance both the parameters.

The EMCC method uses control frame for transmitting and receiving the data between the mobile device and the cloud. The format of the frame is show in the Fig. 4.

\begin{tabular}{|c|c|c|}
\hline$C T$ & $C M$ & $D A T A$ \\
\hline
\end{tabular}

Fig. 4. Control frame of $E M C C$

Here, the CT indicates the compression type, which is a one-bit binary number. The binary ' 0 ' represents no compression and ' 1 ' for the compression. The $C M$ indicates the compression method, which is a two-bit binary number. The following table shows various compression methods and their bit representations used in the EMCC algorithm.

Table- II: $C M$ bits in $E M C C$

\begin{tabular}{|c|l|}
\hline$C M$ Bits & Compression Method \\
\hline 00 & $R L E$ compression method \\
01 & Huffman Encoding Compression \\
10 & $D C T$ \\
11 & $D W T$ \\
\hline
\end{tabular}

Implementing this control frame in $E M C C$ is useful for synchronizing the sender and receiver in the communication system. In other words, the transmitter and receiver end can synchronize on whether the compression exists and the type of compression used, so that the specific decompression process can be used at the receiver side.

\section{Simulation}

The simulation work was carried out by writing the code with the necessary network parameters. In the simulation, we focus on calculating the energy consumption for the mobile end. To differentiate and compare traditional MCC (TMCC) methodology, the proposed model is named as Energy efficient Mobile Cloud Computing (EMCC). The energy consumption model considered for this simulation is based on Mica 2 model [18]. The transmission energy consumption $(E T x)$ and receiving energy consumption (ERx) are 4.602 $\mu \mathrm{J} /$ bit and $2.34 \mu \mathrm{J} /$ bit, respectively. In the simulation setup, we have considered 20 mobile devices and 5 data centres in the cloud with high storage, computation, and battery resources. We have calculated the total energy consumption at the mobile end for both the proposed model EMCC and the traditional model TMCC. The total energy consumption for 20 mobile nodes with 120 iterations is calculated, see the table III. From the table, we say that the proposed model consume less energy compared to the traditional model. This happens because the EMCC compresses the data and the computation to be transmitted from the mobile device to the cloud.

Table- III: Simulation results

\begin{tabular}{|r|r|l|}
\hline $\begin{array}{r}\text { Data } \\
\text { size } \\
(K B)\end{array}$ & $\begin{array}{r}\text { Energy Consumption } \\
\text { using } T M C C \text { for 20 nodes } \\
\text { and 120 rounds }\end{array}$ & $\begin{array}{l}\text { Energy Consumption } \\
\text { using EMCC for 20 } \\
\text { nodes and 120 rounds }\end{array}$ \\
\hline 4 & 407.90 & 284.85 \\
\hline 6 & 612.96 & 400.17 \\
\hline 8 & 815.84 & 482.87 \\
\hline 10 & 1019.81 & 875.05 \\
\hline 12 & 1223.75 & 941.47 \\
\hline
\end{tabular}

\section{Conclusion}

In this paper, we investigated the energy consumption issues in $M C C$. We proposed a method to reduce energy consumption in $M C C$. We have considered two cases for saving the energy in $M C C$ : reducing the size of data and reduce size of the process. As the size of data is reduced, the energy consumption during the communication is reduced. The simulation results show that the proposed method reduces the energy consumption compared to the traditional $M C C$.

\section{REFERENCES}

1. P. M. Mell, and T. Grance, "The NIST Definition of Cloud Computing," Technical Report. NIST, 2011. SP 800-145. Gaithersburg, MD, United States.

2. A. Goyal, and S. Dadizadeh, "A survey on cloud computing," University of British Columbia Technical Report for CS 508, 2009, pp. 55-58.

3. K. Akherfi, M. Gerndt, and H. Harroud, "Mobile cloud computing for computation offloading: Issues and challenges," Applied Computing and Informatics, vol. 14, no. 1, 2018, pp 1-16.

4. T. H. Noor, S. Zeadally, A. Alfazi, and Q. Z. Sheng, "Mobile cloud computing: Challenges and future research directions," Journal of Network and Computer Applications, vol. 115, 2018, pp. 70-85.

5. T. Le Vinh, R. Pallavali, F. Houacine, and S. Bouzefrane, "August. Energy efficiency in mobile cloud computing architectures," In 4th International Conference on Future Internet of Things and Cloud Workshops (FiCloudW), 2016, pp. 326-331.

6. E. Benkhelifa, T. Welsh, L. Tawalbeh, Y. Jararweh, A. Basalamah, "User profiling for energy optimization in mobile cloud computing," Procedia Computer Science, vo. 52, 2015, pp. 1159-1165.

7. W. Zhang, Y. Wen, K. Guan, D. Kilper, H. Luo, and D. O. Wu, "Energy-optimal mobile cloud computing under stochastic wireless channel," IEEE Transactions on Wireless Communications, vol. 12, no. 9, 2013, pp. 4569-4581.

8. K. Gai, M. Qiu, H. Zhao, L. Tao, and Z. Zong, "Dynamic energy-aware cloudlet-based mobile cloud computing model for green computing," Journal of Network and Computer Applications, vol. 59, 2016, pp. 46-54.

9. D. Huang, T. Xing, and H. Wu, "Mobile cloud computing service models: a user-centric approach," IEEE Network, vol. 27, no. 5, 2013, pp. 6-11.

10. A. P. Miettinen, and J. K. Nurminen, "Energy Efficiency of Mobile Clients in Cloud Computing," HotCloud, vol. 10, 2010, pp. 4-4,2010.

11. S. Robinson, "Cellphone energy gap: Desperately seeking solutions," Strategy Analytics, 2009.

12. T. Le Vinh, R. Pallavali, F. Houacine, and S. Bouzefrane, "Energy Efficiency in Mobile Cloud Computing Architectures," In Future Internet of Things and Cloud Workshops (FiCloudW), IEEE International Conference on (pp. 326-331). 2016, August.

13. J. Abel, and W. Teahan, "Universal Text Preprocessing for Data Compression," IEEE Trans. Comput., vol. 54, no. 5, 2005, pp. 497-507.

14. A. Raju, and N. Singh, "A Review on data compression techniques," International Journal of Applied Research in Computer Science and Software Engineering (IJARCSSE), vol. 5, 2015.

15. N. Sharma, and J. Singh, "A review on various lossless text data compression techniques," 
International journal of electrical science, vol. 12, 2014, pp. 58-63.

16. P. Kavitha, "A Survey on Loassless and Lossy Data Compression Methods," International Journal of Computer Science \& Engineering Technology (IJCSET), vol. 7, 2015, pp. 110-114.

17. M. Hosseini, "A Survey of Data Compression Algorithms and Their Applications," Applications of Advanced Algorithms, Simon Fraser University, Canada, Jan 2012.

18. V. Shnayder, M. Hempstead, B. Chen, G. Werner Allen, and M. Welsh, "Simulating the Power Consumption of LargeScale Sensor Network Applications," SenSys'04, Baltimore, Maryland, USA, November 3-5, 2004.

\section{Authors ProfiLe}

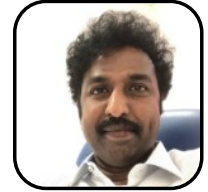

communications

D Satyanarayana obtained his bachelor, master, and doctorate degrees in computer engineering specialization. Currently he is working as an assistant professor in College of Engineering, University of Buraimi, Oman, since 2012. His research areas include wireless communications, sensor networks, and visible light

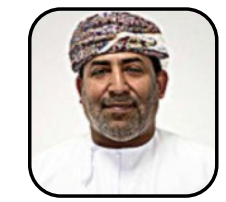

Abdullah Said Al Kalbani obtained his bachelore, master, and doctorate degrees in computer engineering specialization. Currently he is working as a Dean in College of Engineering, University of Buraimi, Oman. His research areas include Network Security, Data Security, sensor networks, and wireless communications. 